\title{
A Measurement System for Complex Optical Investigations of Erosion Laser Jets
}

\author{
V. K. Goncharov and K. V. Kozadaev* \\ Sevchenko Research Institute of Physical Problems, Belarusian State University, ul. Kurchatova 7, Minsk, 220108 Belarus \\ *e-mail: kozadaeff@mail.ru \\ Received November 2, 2013; in final form, March 19, 2014
}

\begin{abstract}
A measurement system for implementing a technique of high spatiotemporal and spectral resolutions is proposed for complex investigations of processes of the formation and evolution of laser-induced bunches under normal atmospheric conditions. Such optical techniques for studying laser-induced metal plasma as laser probing and spectral analysis are used in the diagnostics of plasma parameters. The use of this system made it possible to obtain original data on laser erosion of metals exposed to nanosecond pulses.
\end{abstract}

DOI: $10.1134 / \mathrm{S} 0020441214060062$

\section{INTRODUCTION}

The technologies for obtaining high-density laser radiation within narrow spatial, time, and spectral intervals opened new prospects for the development of methods for precision surface processing of materials, contactless diagnostics of high-speed processes, highprecision measurements, and information transfer. The conventional advantages of laser material processing are as follows: locality of the action, minimum deformations of a material, a high concentration of the applied energy, the absence of a mechanical contact with the processes object, a high degree of automation, the ecological purity of processing, and a high working capacity. However, during the action of intense laser pulses on metals, a vapor-plasma jet is usually produced, which contains products of damage of a processed material. The appearance of an erosion laser jet and its interaction with incident radiation impair the conditions for the laser-energy transmission to the surface of the processed material, thus reducing the controllability of the entire processing procedure [1-3].

In connection with this, studying the energy, time, spatial, and spectral characteristics of vapor-plasma bunches, which appear under intense laser irradiations, are of great practical interest. This paper describes a specialized automated hardware and software measurement system that provides a technique of high spatiotemporal and spectral resolutions. Here, the diagnostics of the parameters of an erosion laser jet (ELJ) is performed using several research techniques: laser probing, monitoring of the effective diameter and concentration of particles in the condensed phase, and the spectral analysis of the ELJ.

\section{LASER-PROBING TECHNIQUE FOR AN ELJ}

One of the main experimental approaches to investigating the dynamics of the optical characteristics of ELJs in this system is to use the technique of transverse probing of an erosion jet by radiation of an auxiliary laser [4]. The essence of this method consists in the parallel recording of the components (incident, transmitted, and scattered) of a probing pulse during its interaction with the ELJ. The results of these measurements allow one to determine the dynamics of the total loss of the probing radiation in the ELJ, as well as the dynamics of the scattering and absorption losses in the ELJ. The losses are calculated on the basis of the balance of the probing-radiation components.

The choice of the auxiliary-laser radiation wavelength is determined by the possibility of reliable spectral separation of a probing optical signal and an acting pulse. In this case, the probing-radiation power density, on the one hand, must be sufficient for reliable detection of the scattered component (which may be $<1 \%$ of the incident component) and, on the other hand, must not perturb the investigated medium. In this study, we experimentally established the optimal range of the power densities of probing radiation: $10^{4}-10^{5} \mathrm{~W} / \mathrm{cm}^{2}$. One of the main advantages of the laser-probing method is the possibility of obtaining information on the processes that occur in an ELJ in real time with a high spatial resolution (for a typical duration of plasma processes of $\sim 10^{-6} \mathrm{~s}$ ).

When radiation is incident on an arbitrary semitransparent medium in the absence of nonlinear effects, a fraction of the incident radiation passes through this medium, another fraction is absorbed, and the remaining part is scattered by the medium [5]. The equation for probing radiation can be written as

$$
P_{\text {inc }}(t)=P_{\mathrm{tr}}(t)+P_{\mathrm{sc}}(t)+P_{\mathrm{abs}}(t),
$$




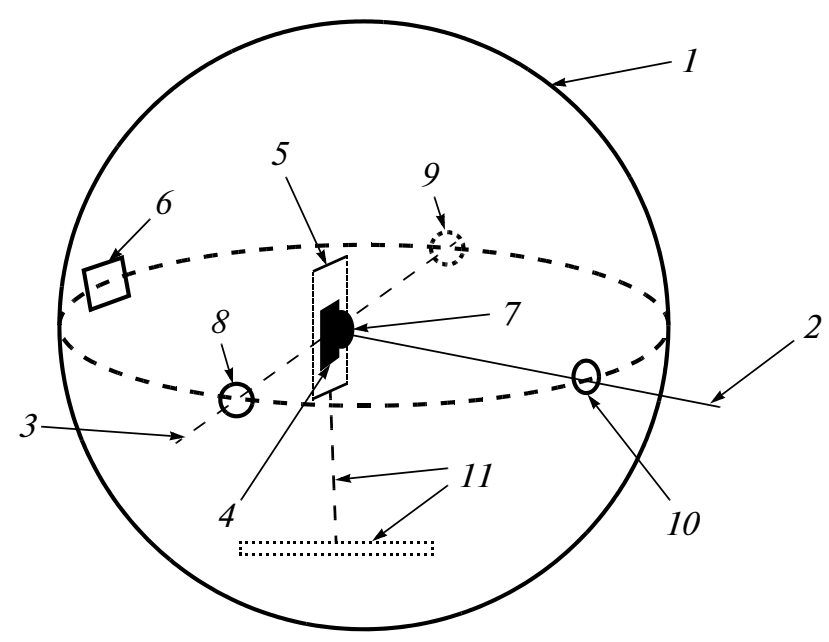

Fig. 1. General scheme of laser probing: (1) integrating sphere, (2) acting laser pulse, (3) probing laser pulse, (4) laser target, (5) target fixation system, (6) PMT window, (7) ELJ, $(8,9)$ input and output holes for probing radiation, respectively, (10) input hole for acting radiation, and (11) base of the target fixation system.

where $P_{\text {inc }}(t)$ is the incident-radiation power, $P_{\mathrm{tr}}(t)$ is the transmitted-radiation power, and $P_{\mathrm{sc}}(t)$ and $P_{\mathrm{abs}}(t)$ are the powers of scattered and absorbed radiations.

In relative units, Eq. (1) has the form

$$
K_{\mathrm{tr}}(t)+K_{\mathrm{sc}}(t)+K_{\mathrm{abs}}(t)=1,
$$

where

$$
\begin{gathered}
K_{\mathrm{tr}}(t)=\frac{P_{\mathrm{tr}}(t)}{P_{\mathrm{inc}}(t)} ; \quad K_{\mathrm{sc}}(t)=\frac{P_{\mathrm{sc}}(t)}{P_{\mathrm{inc}}(t)} ; \\
K_{\mathrm{abs}}(t)=\frac{P_{\mathrm{abs}}(t)}{P_{\mathrm{inc}}(t)} .
\end{gathered}
$$

Thus, by measuring the values of $P_{\text {inc }}(t), P_{\mathrm{tr}}(t)$, and $P_{\mathrm{sc}}(t)$ and using formulas (1) and (2), one can determine the transmission, scattering, and absorption coefficients for the interaction of probing radiation with an ELJ.

The absorption and scattering energy losses can be separated in practice, if the target is placed at the center of an integrating sphere; this allows simultaneous monitoring of the power irradiating the erosion jet and the jet-transmitted and scattered components of probing radiation (i.e., the actual transmission, scattering, and absorption coefficients for the ELJ in the region of probing radiation-jet interaction) [4].

A general diagram of laser probing is shown in Fig. 1. High-power laser pulse 2 is directed through hole 10 to metal target 4 in the form of a flat plate. As a result of an effect of radiation on the target material, ELJ 7 is produced. Its transverse probing is concurrently performed with pulse 3 of an auxiliary ruby laser. Probing radiation is injected and extracted through holes 8 and 9 , respectively. ELJ-scattered probing-pulse radiation is uniformly distributed over the entire inner scattering surface of integrating sphere 1 . Thus, by recording a signal from a small area of sphere 6 and performing an appropriate calibration, it is possible to determine the scattered-component value as a whole. For this purpose, a photomultiplier tube (PMT), which is protected with a set of broadband and interference spectral filters against the influence of extraneous illuminations and acting radiation, is installed in window 6 .

Such an experimental scheme has a number of technical advantages. Universal holder 5 provides convenient placement of targets with various shapes, thus allowing one to probe not only ELJs of different materials, but also optically transparent condensed ultradisperse media (colloid solutions, films, etc.). The possibility of smoothly precisely changing the height of the auxiliary beam above the target surface of the ELJ provides a rather high spatial resolution of probing (down to $0.1 \mathrm{~mm}$ ). Depending on the objective of the experiment, the probing-radiation beam diameter can be also varied (from 0.1 to $8 \mathrm{~mm}$ ) $[4,6,7]$.

\section{CALCULATION OF THE CHARACTERISTICS OF THE CONDENSED METAL PHASE IN PLASMA}

As was shown in the Mie theory [8], the scattering and extinction efficiency factors $Q_{\mathrm{sc}}$ and $Q_{\mathrm{ext}}$ of probing radiation for homogeneous monodisperse spherical metal particles can be represented in the form of infinite series with complex coefficients $a_{n}$ and $b_{n}$ that depend on the particle material and the diffraction parameter $x=\frac{\pi d}{\lambda}$ :

$$
\begin{aligned}
& Q_{\mathrm{sc}}=\frac{2}{x^{2}} \sum_{n=1}^{\infty}(2 n+1)\left(\left|a_{n}\right|^{2}+\left|b_{n}\right|^{2}\right) ; \\
& Q_{\mathrm{ext}}=\frac{2}{x^{2}} \sum_{n=1}^{\infty}(2 n+1) \operatorname{Re}\left(a_{n}+b_{n}\right),
\end{aligned}
$$

where $d$ is the particle size, $\lambda$ is the probing-radiation wavelength, and the absorption efficiency factor is $Q_{\text {abs }}=Q_{\text {ext }}-Q_{\text {sc }}$.

These efficiency factors are theoretically estimated using the known calculation scheme proposed in $[8,9]$. To illustrate this approach, only some key relationships of this technique are presented in this paper.

The coefficients $a_{n}$ and $b_{n}$ are determined through the Riccati-Bessel functions $\psi_{n}$ and $\xi_{n}$ and their derivatives:

$$
\begin{aligned}
& a_{n}=\frac{m \psi_{n}(m x) \psi_{n}^{\prime}(x)-\psi_{n}(x) \psi_{n}^{\prime}(m x)}{m \psi_{n}(m x) \zeta_{n}^{\prime}(x)-\zeta_{n}(x) \psi_{n}^{\prime}(m x)} ; \\
& b_{n}=\frac{\psi_{n}(m x) \psi_{n}^{\prime}(x)-m \psi_{n}(x) \psi_{n}^{\prime}(m x)}{\psi_{n}(m x) \zeta_{n}^{\prime}(x)-m \zeta_{n}(x) \psi_{n}^{\prime}(m x)},
\end{aligned}
$$

where $m$ is the complex refractive index of the medium; $\rho$ is the argument equal to $x$ or $m x$ depending on the function in which it is used: $\psi_{n}(\rho)=\rho j_{n}(\rho)$, 
$\xi_{n}(\rho)=\psi_{n}(\rho)-i \chi_{n}(\rho), \chi_{n}(\rho)=\rho y_{n}(\rho)$, and the spherical Bessel functions $j_{n}(\rho)$ and $y_{n}(\rho)$ are expressed through the Bessel functions of the first and second kind: $J_{n+1 / 2}(\rho)$ and $Y_{n+1 / 2}(\rho)[9]$.

To perform the subsequent calculations, it is convenient to introduce the logarithmic derivative of the function $\psi$ :

$$
D_{n}(\rho)=\frac{d}{d \rho} \ln \psi_{n}(\rho)=\frac{\psi_{n}^{\prime}(\rho)}{\psi(\rho)} .
$$

This yields

$$
\begin{gathered}
a_{n}=\frac{\left[\frac{D_{n}(m x)}{m}+\frac{n}{x}\right] \psi_{n}(x)-\psi_{n-1}(x)}{\left[\frac{D_{n}(m x)}{m}+\frac{n}{x}\right] \xi_{n}(x)-\xi_{n-1}(x)} ; \\
b_{n}=\frac{\left[m D_{n}(m x)+\frac{n}{x}\right] \psi_{n}(x)-\psi_{n-1}(x)}{\left[m D_{n}(m x)+\frac{n}{x}\right]+\xi_{n}(x)-\xi_{n-1}(x)} .
\end{gathered}
$$

As a result, we obtain the recurrence formulas for $D_{n}$ [4]:

$$
D_{n-1}(\rho)=\frac{n}{\rho}-\frac{1}{D_{n}(\rho)+\frac{n}{\rho}} .
$$

Two calculation schemes using (11) are possible for the functions $D_{n}(\rho)$ : the forward recursion (higher orders are obtained from lower ones) and the reverse recursion (lower orders are obtained from higher ones). It was shown in [10] that the calculation of $D_{n}$ is stable with respect to the reverse recursion, i.e., if we can estimate the value of $D_{N}$ for a certain large $N$ with a certain accuracy, then using the reverse recursion with the help of (11), all $D_{n}$ for $n<N$ can be calculated with an accuracy better than the initial accuracy for $D_{N}$.

Although series (4) and (5) are infinite, it was shown in [9] that it is sufficient to consider only a finite number $M$ of terms in these series in a numerical calculation. Here, $M$ is the nearest integer with respect to $M_{x}=x+4 x^{1 / 3}+2$, where $x$ is the diffraction parameter. The functions $D_{n}(m x)$ in (9) and (10) are calculated from (11) via the reverse recursion beginning with $n=N$. Provided that $N$ is appreciably larger than $M$ and $|m x|$, the functions $D_{n}(m x)$ with numbers $n<M$ almost do not depend on the choice of $D_{n}(m x)$, and $D_{n}(m x)$ for large $n$ are small. Therefore, we can chose $N=\max (M,|m x|)+15$ and assume that $D_{N}=o+i o$.

Finally, we obtain the resulting calculation scheme. Using the values of $x$ and $m, \psi_{n}$ and $\chi_{n}$ are calculated, beginning with $\psi_{-1}(x)=\cos x, \psi_{0}(x)=\sin x$, and $\chi_{-1}(x)=-\sin x, \chi_{0}(x)=\cos x$, up to $n=M$. Then, (11) is used to calculate all $D_{n}$ for $n<N$, beginning with $D_{N}=o+i o$. Subsequently, $a_{n}$ and $b_{n}$ are determined from (9) and (10), and the loss efficiency factors are determined using (4) and (5). The comparison of the theoretically calculated scattering and absorption efficiency factors to the experimentally measured scattering and absorption coefficients allows one to estimate the diameter of particles in an ELJ [4].

Along with the particle diameter, the numerical and volume concentrations of particles in the liquiddrop phase are also important characteristics of an erosion jet. According to the Bouguer law,

$$
I=I_{0} e^{-\tau},
$$

where $I$ is the intensity of transmitted radiation, $I_{0}$ is the intensity of incident radiation, and $\tau$ is the optical density of the medium.

Then,

$$
K_{\mathrm{tr}}=I / I_{0}=e^{-\tau}
$$

and

$$
K_{\mathrm{sc}}+K_{\mathrm{abs}}=1-e^{-\tau}
$$

Since the value of $\tau$ in our experiments is low [11], the exponent $e^{-\tau}$ can be expanded into a Taylor series:

$$
e^{-\tau}=1-\frac{\tau}{1 !}+\frac{\tau^{2}}{2 !} \ldots \approx 1-\tau
$$

Substituting (15) into equality (14), we obtain

$$
K_{\mathrm{sc}}+K_{\mathrm{abs}}=\tau \text {. }
$$

For a single scattering event,

$$
\tau=Q_{\text {ext }} \pi d^{2} N_{c} l / 4
$$

where $N$ is the numerical particle concentration and $l$ is the length of interaction of probing radiation with erosion products.

On the basis of (17), the expression for the numerical concentration value is obtained:

$$
N_{c}=\frac{4\left(K_{\mathrm{sc}}+K_{\mathrm{abs}}\right)}{\pi d^{2} Q_{\mathrm{ext}} l}
$$

Using the known values of $N$ and particle dimensions, we can determine the volume concentration $C_{V}$ of particles in the liquid-drop phase (the ratio of the volume occupied by particles in the condensed phase in the studied zone to the entire volume of the studied zone). This characteristic is an important parameter in the quantitative evaluation of the obtained results. The volume concentration $C_{V}$ can be determined in the Rayleigh approximation from the formula

$$
C_{\mathrm{V}}=\frac{1}{6} \pi d^{3} N_{c}
$$

Substituting (19) into (18) yields

$$
C_{\mathrm{V}}=\frac{2}{3} \frac{K_{\mathrm{sc}}+K_{\mathrm{abs}}}{Q_{\mathrm{exc}} l} d
$$

Note that the above-described technique for calculating the coefficients is valid only for a single scattering event at monodisperse particles, when $Q_{\mathrm{sc}} / Q_{\mathrm{abs}}=$ $K_{\text {sc }} / K_{\text {abs }}$. It was established in [12] that for particle diameters of $<0.15 \mu \mathrm{m}$, the medium polydispersity 
does not significantly affect the ratio of the absorption and scattering factors.

The single-scattering condition is fulfilled if the mean free path $l^{\prime}$ of a scattered photon is larger than the size of a probed object $l$; i.e., $l^{\prime}=\frac{1}{N_{c} \sigma_{\mathrm{sc}}}>l$, where $N$ is the particle concentration and $\sigma_{\mathrm{sc}}=\pi d^{2} Q_{\mathrm{sc}} / 4$ is the effective scattering cross section. The following limitation on the particle concentration is obtained from this expression: $N_{c}<\frac{4}{\pi d^{2} Q_{\mathrm{sc}}}$. For a typical diameter $d \sim 0.08 \mu \mathrm{m}, Q_{\mathrm{sc}} \sim 0.07$; hence, $N_{c}<10^{12} \mathrm{~cm}^{-3}$.

Thus, the scheme of calculation of the diameters and concentrations of the liquid-drop phase of an ELJ for each time moment is as follows.

(1) The laser-probing technique is used to experimentally determine the probing-radiation scattering and absorption coefficients in an ELJ.

(2) The particle diameters can be calculated on the basis of comparison of the scattering and absorption efficiency factors, which were theoretically compared according to the Mie theory, to the experimentally measured probing-radiation scattering and absorption coefficients. The concentration of particles in the liquid-drop phase in an ELJ can be calculated from the known values of the diameters and the probed-zone width.

The accuracy in determining the diameter of tungsten particles that are formed in an ELJ was evaluated in [13] using the laser-probing method. For this purpose, tungsten ELJs were probed in several experiments. Subsequently, the formed particles of the liquid-drop phase of this metal were deposited on a substrate, which was placed in a chamber of an electron microscope. Processing of the obtained images made it possible to determine the size distribution profile for ensembles of particles. In particular, it was established that the particle-size distribution profile is similar to a $\Gamma$ distribution to a maximum degree, and its mathematical expectation corresponds to the evaluation of the particle diameters by the laser-probing method with a high accuracy (error of $\sim 5-7 \%$ ).

\section{SPECTRAL DIAGNOSTICS OF AN ELJ}

An intense laser pulse that irradiates a target (in the form of a solid, liquid, gas, or aerosol) may initiate dissociation, excitation, and ionization processes of molecules and atoms of a substance. This leads to the appearance of a plasma cloud that expands in the direction of an external medium (vacuum or foreign gases depending on the experimental conditions). As a result of the interaction of laser radiation with the material, several physical processes can be observed: material ablation, pulsed target acceleration, and emission of high-energy particles and electromagnetic radiation within a range from radio waves to $\mathrm{X}$ rays. All these phenomena are applied in practice.

In some studies, the emission spectra of erosion plasma are studied in a wavelength range of 200$1100 \mathrm{~nm}$. Such investigations are called laser-induced plasma spectroscopy (LIPS). As a whole, this is the group of spectroscopic methods that are intended for studying the properties of laser plasma and proximate analysis of the elemental compositions of a substance in any aggregate state via analysis of the emission spectra of laser-induced plasma. The LIPS method has such advantages as the high spatial resolution (owing to the possibility of precisely focusing the laser beam), the absence of strict requirements for thorough preparation of the sample surface, and the possibility of conducting remote investigations under difficult conditions (for example, during melting or burning of samples). In this study, the LIPS technique is used to determine the parameters of laser plasma of metals [14].

Laser-induced plasma has some specific features as compared to plasma generated via other physical effects (e.g., electric-discharge plasma, etc.). Because the case of vapor outflow to a half-space is realized under the action of laser radiation on a metal, the forming plasma cloud is characterized by a large inhomogeneity (this effect is more pronounced during interaction of laser plasma with radiation by which it was initiated). As a rule, pulsed laser radiation is used, thus leading to considerable plasma nonstationarity (especially when short and ultrashort pulses are used). Correspondingly, when the results of investigations using the LIPS technique are interpreted, it is necessary to take the spatiotemporal structure of the plasma cloud into account. When measurements are conducted in the atmosphere of dense gases, this cloud may have a quite complex configuration and small transverse dimensions. Nevertheless, depending on the experimental conditions, either a local thermodynamic equilibrium or a partial local thermodynamic equilibrium is used with some reservations as the main theoretical models of the plasma state in LIPS.

The advent of the experimental equipment that provides a high temporal resolution of optical processes (generation of short and ultrashort laser pulses, fast analog-to-digital conversion, high-sensitivity photodetectors) opened new possibilities for investigating fast transient processes. In this study, elements of time-resolved laser spectroscopy are used to analyze the dynamics of the spectral structure of individual lines of metals under study [15].

\section{STRUCTURE OF THE MEASUREMENT SYSTEM FOR COMPLEX STUDIES OF THE OPTICAL CHARACTERISTICS OF AN ELJ}

A schematic diagram of the research complex is shown in Fig. 2. It contains the following basic units: (I) a unit for the formation of acting pulses and 
recording of their characteristics, (II) a unit for studying the spectral characteristics of an ELJ, (III) a unit of laser probing of an ELJ, (IV) a unit for experimental-data processing, and $(V)$ synchronization unit.

Unit $I$ is intended for forming an acting pulse and recording its characteristics and includes laser facility 1 , deflecting plates 2 and 3, pulse-shape recorder 4 (ФД $21 \mathrm{КП),} \mathrm{and} \mathrm{calorimeter} \mathrm{5.} \mathrm{The} \mathrm{proposed}$ arrangement of the complex allows application various laser facilities as sources of acting pulses. However, this study was performed using ГОС 1001 and LS 2137 neodymium lasers $(\lambda=1064 \mathrm{~nm})$ (http://www.lotis-tii. $\mathrm{com} / \mathrm{rus} /$ productid21.php) that generate pulses in the $\mathrm{Q}$-switched mode. These laser facilities make it possible to generate pulses with different durations (20 and $100-200 \mathrm{~ns})$ and a high power density $\left(10^{8}-10^{9} \mathrm{~W} / \mathrm{cm}^{2}\right)$ at a rather large cross-sectional size of the focusing spot $(1-3 \mathrm{~mm})$. Experiments showed that the reproducibility of the results of individual experiments are mainly determined by the accuracy of reproducing the shape of the influencing pulses and their energy parameters (other conditions being the same). Modern laser facilities (including LOTIS 2137) meet this requirement to the highest degree; in other cases, to confirm the reliability of the obtained estimates, it is necessary to collect statistical data by performing several tens of experiments.

The main purpose of unit $I I$ for studying the spectral characteristics of ELJs is the implementation of the LIPS analytical method. Studying the dynamics of integral ELJ glow may be also of practical interest [15]. These research functions are experimentally provided by a spectrophotometer (MS 2001i, http://www. solartii.ru/spectral_instruments/ms200.htm) with CCD array 13 as an optical sensor; monochromator 14 (МДР-12) joined to silicon PMT 15 (SPM MicroSL10020-X13 SensL, http://sensl.com/products/siliconphotomultipliers). PMTs of this type are characterized by a rather high gain $\left(\sim 10^{6}\right)$ within a range of 350$900 \mathrm{~nm}$, a low dark-noise level $(0.1 \mu \mathrm{A})$ in comparison to analogous sensors, and a short cell-recovery time (30 ns). Integral-glow sensor 10 (ФД $21 \mathrm{K \Pi )} \mathrm{is}$ equipped with interference mirror $11(\lambda=1064 \mathrm{~nm})$ for eliminating a spurious contribution of influencing radiation. Moreover, the unit contains system 7-9 for directing radiation to the surface of target 17 and optical systems 12 and 16 for forming ELJ image 18 at the entrances of the spectral instruments.

The laser-probing technique for ELJs is used to study the ELJ-formation and further-evolution processes. Unit III that provides these functions implements the probing scheme (Fig. 1) and contains system $21,22,25,26$ for forming and directing a probing pulse to the ELJ, system $6,7,19$ for directing the jetinducing pulse to target 31 , and a system for recording the scattered and absorbed probing-radiation components, which consists of ФД 21 КП photodiodes $(24,27)$ and an SPM MicroSL-10020-X13 SensL PMT (29). Depending on the aim of the experiment, a ruby laser $(\lambda=694.3 \mathrm{~nm})$ and a semiconductor laser $(\lambda=$ $800 \mathrm{~nm}$ ) were used. In this case, the optical sensors are protected against the effect of power radiation and foreign flashes by interference filters 23 and 28 at the wavelength of probing radiation.

Unit $I V$ for experimental-data processing includes a multichannel digital oscilloscope 33 (B432, http:// auris.ru/rus/products.htm) with a sampling frequency of $100 \mathrm{MHz}$, thus allowing one to attain a time resolution of $\sim 10 \mathrm{~ns}$, and personal portable computer 32 . Software that provides automation of the recording, primary-processing, and experimental-data-representation processes was developed for the main operating modes of the research complex.

Synchronization unit $V$ is a multichannel generator of delayed pulses 34 (G 200P, http://www.spetspribor. info/products/laboratory/g-200p.html) that allows formation of triggering pulses in wide ranges of their durations and time delays individually for each channel. In addition, to eliminate situations of emergency acting-laser firing by induced interferences, opticalisolation scheme 35 is used for this triggering channel [16].

\section{OPERATING MODES OF THE EXPERIMENTAL COMPLEX}

The measurement system is intended for operating in two main modes.

(1) The mode of studying the dynamics of the spatial and phase structures of ELJs for metals. Laser target 31 (a plane-parallel plate of a studied material) is fixed at the center of integrating sphere 20 , as shown in Fig. 2. An acting pulse that is formed with laser facility 1 is focused to the surface of target 31 using rotating system 6, 7 and lens 19. By changing the distance from lens 19 to target 31 , it is possible to provide different power-density levels of acting radiation: from $10^{7}$ to $10^{10} \mathrm{~W} / \mathrm{cm}^{2}$. The action of a laser pulse results in the formation of ELJ 30, which, beginning with its onset moment, is probed with radiation of auxiliary laser 25 . Measuring the ratios of the incident, transmitted, and scattered components of probing radiation allows determination of the ELJ phase-structure dynamics (including the parameters of the ELJ condensed phase) with a high time resolution (no worse than $10 \mathrm{~ns}$ ). The measured characteristics are digitized using multichannel oscilloscope 33 and automatically processed with portable computer 32 .

(2) The mode of studying the dynamics of the spectral structure of glow from ELJs of metals. An acting pulse is focused to the surface of target 17 using rotating system 7,8 and lens 9 . In this case, it is also possible to change the power density of acting radiation from $10^{7}$ to $10^{10} \mathrm{~W} / \mathrm{cm}^{2}$. Using systems 12 and 16 , an image of the forming ELJ is transferred to the input of spectral instruments 13-15. Spectrophotometer 13 allows investigation of the time-integral ELJ glow spectrum with a high spectral resolution (up to $0.1 \mathrm{~nm}$ ). 


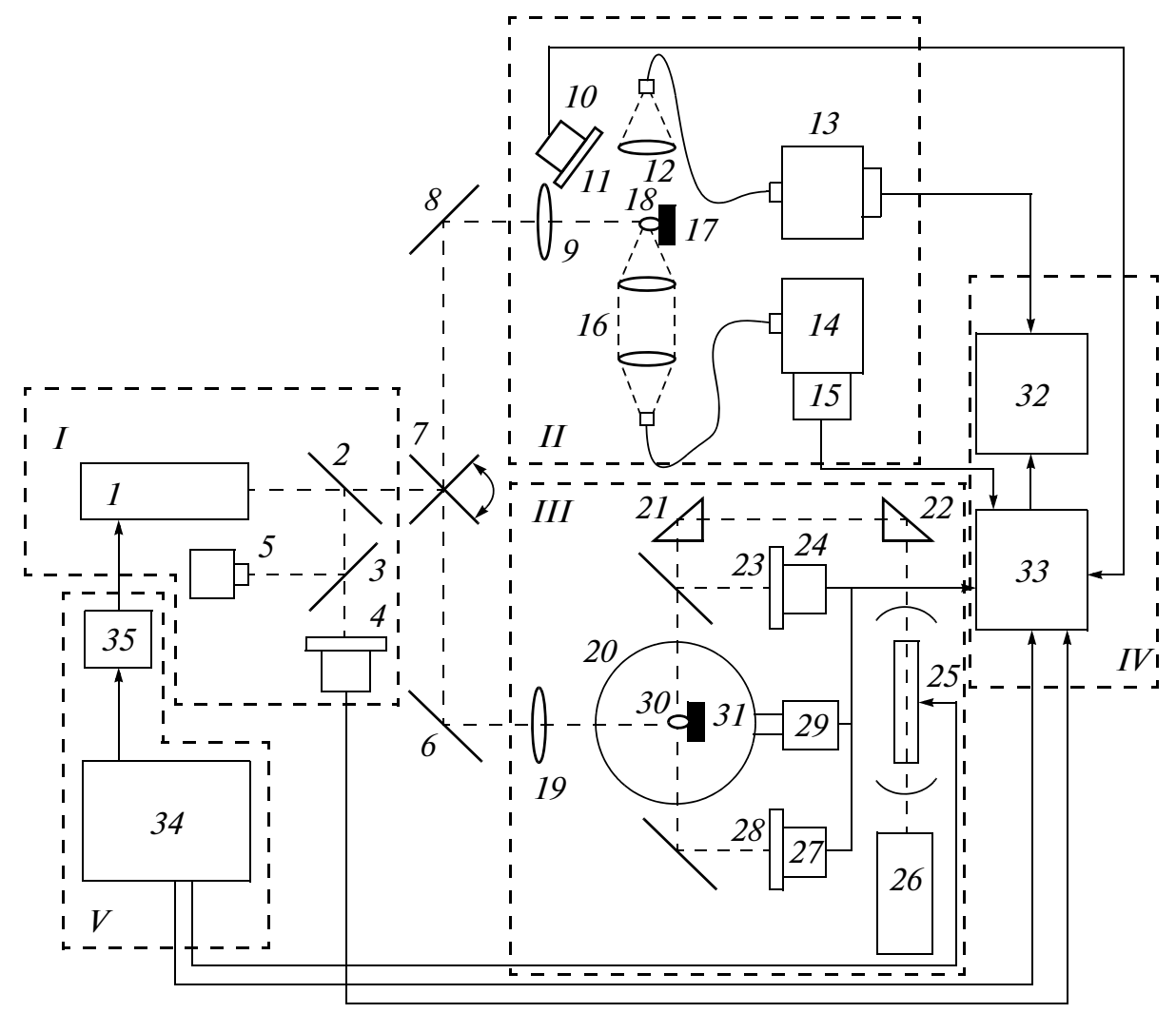

Fig. 2. Schematic diagram of the research complex: $(I)$ unit for the formation of acting pulses and recording their characteristics, (II) unit for studying the spectral characteristics of an ELJ, (III) unit of laser probing of an ELJ, (IV) unit for experimental-data processing, ( $V$ synchronization unit. (1) Acting laser, $(2,3)$ deflecting transparent plates, (4) acting-pulse shape recorder, (5) calorimeter of the acting-pulse energy, $(6-8)$ mirrors, $(9,19)$ lenses for focusing acting radiation, $(10)$ sensor of integral ELJ glow, $(11,23,28)$ optical filters, $(12,16)$ optical systems forming ELJ images at the entrances of spectral instruments, (13) spectrophotometer, (14) monochromator, $(15,29)$ PMT, $(17,31)$ laser targets, $(18,30)$ ELJ, (20) integrating sphere, $(21,22)$ rotating prisms, $(24,27)$ recorders of the probing-radiation intensity, (25) probing laser, (26) auxiliary alignment laser, (32) portable computer, (33) multichannel analog-to-digital converter (ADC), (34) generator of sync pulses, and (35) system for optical isolation of acting-laser triggering.

Monochromator 14, which is joined to PMT 15, provides studies of the glow dynamics in narrow spectral ranges (with a width of $0.5 \mathrm{~nm}$ ) of ELJs with a high time resolution (no worse than $10 \mathrm{~ns}$ ). Sensor 10 allows investigations of the spectrum-integrated glow dynamics for ELJs for metals with a high time resolution (no worse than $10 \mathrm{~ns}$ ). The measured characteristics are also digitized with multichannel oscilloscope 33 and automatically processed with portable computer 32.

\section{RESULTS}

Owing to the complex approach to the accomplishment of experiments and the possibility of varying both the modes of laser action and probing pulses, the developed research complex allowed us to obtain several original results when studying the interaction of intense laser radiation with metals under atmospheric pressure.

Experiments showed [16] that under the action of submicrosecond pulses ( $200 \mathrm{~ns})$ with a high power density $\left(10^{8}-10^{9} \mathrm{~W} / \mathrm{cm}^{2}\right)$, an intensely glowing plasma bunch forms in the near-surface region of the target $\sim 50 \mathrm{~ns}$ after the laser-action onset (this time is compared to the acting-pulse rise time). The laser jet propagates in the direction of an external medium and absorbs almost the entire energy of the trailing edge of the acting laser pulse owing to the inverse braking effect and, as a result, the jet is considerably heated.

The characteristic propagation speed of the plasma bunch at the beginning is $7-20 \mathrm{~km} / \mathrm{s}$ depending on the type of a metal target. After the laser-action intensity decreases, the jet slowly cools mainly at the expense of the adiabatic plasma expansion. This can be judged by a certain delay of the jet-glow attenuation with respect to the trailing edge of the acting laser pulse. In this case, a scattered component appears in the structure of the probing-radiation extinction, thus indicating the formation of local plasma inhomogeneities inside a jet. At this moment, they determine the transmission of probing laser by the jet. Subsequently, 2-2.5 $\mu$ s after the start of the laser action, the probing-radiation absorption processes again begin to prevail over scat- 
tering at a sufficiently low loss level in the jet, thus indicating the onset of fine-disperse drop formation owing to the condensation of the plasma cloud. The investigation results [6] show that the condensedphase expansion continues for a rather long time $(\sim 300-400 \mu \mathrm{s})$ after the action and leads to the appearance of nanoparticles of the processes metal.

A qualitative coincidence with the previous results was demonstrated in studies of analogous processes for $20-n s$ pulses with high power densities [7, 17]. The main differences consist in a certain decrease in the initial velocity of the plasma bunch (to $4-14 \mathrm{~km} / \mathrm{s}$ ) and a substantially larger transparency of the ELJ at the formation stage, especially for a laser-power density of $\sim 10^{8} \mathrm{~W} / \mathrm{cm}^{2}$.

The regularities in the formation of the spectral signal of the characteristic lines of elements in the ELJ were demonstrated. Because exactly these data are used in the diagnostics of the parameters of plasma bunches, the ELJ evolution stages for which the electron-density and temperature characteristics are evaluated should be separated. The appearance of a pronounced line spectrum points to the presence of substantially cooled plasma with quite low values of the electron-density and temperature. The initial processes of the ELJ formation within 200-300 ns after the laser action are characterized by radiation with a continuous spectrum with a wide peak near 400$500 \mathrm{~nm}$ [15].

The performed investigations allowed us to substantiate the efficiency of the process of laser synthesis of metal nanostructures through the condensation of ELJs of metals at atmospheric pressure. On the basis of these materials, new practical techniques for forming metal nanostructured systems for different purposes [18-20].

\section{CONCLUSIONS}

The measurement system described in this paper operates on the basis of optical techniques and allows conducting complex investigations of erosion vaporplasma jets, which appear under the action of intense nanosecond laser pulses on metal targets. The obtained experimental results made it possible to establish the main regularities in the formation and kinetics of the liquid-drop metal phase in the nearsurface target region at atmospheric pressure. The condensation mechanism of the formation of liquid drops, which are produced in ELJs under the action of nanosecond laser pulses, was experimentally discovered and substantiated. The results of these studies underlie new methods for forming metal nanostructures for different purposes.

\section{REFERENCES}

1. Anisimov, S.I., Imas, Ya.A., Romanov, G.S., and Khodyko, Yu.V., Deistvie lazernogo izlucheniya bol'shoi moshchnosti na metally (Action of High Power Laser Radiation on Metals), Moscow: Nauka, 1970.

2. Rykalin, N.N., Uglov, A.A., and Kokora, A.N., Lazernaya obrabotka materialov (Laser Processing of Materials), Moscow: Mashinostroenie, 1975.

3. Mirkin, L.I., Fizicheskie osnovy obrabotki materialov luchami lazera (Physical Foundations of Material Processing by Laser Beams), Moscow: MGU, 1975.

4. Goncharov, V.K., Kontsevoi, V.L., Puzyrev, M.V., and Smetannikov, A.S., Prib. Tekh. Eksp., 1995, no. 5, p. 146.

5. Born, M. and Volf, E., Principles of Optics: Electromagnetic Theory of Propagation, Interference and Diffraction of Light, Cambridge: Cambridge University, 1999; Moscow: Nauka, 1973, 7th ed.

6. Goncharov, V.K. and Kozadaev, K.V., J. Eng. Phys. Thermophys., 2010, vol. 83, p. 90.

7. Goncharov, V.K., Kozadaev, K.V., Shchegrikovich, D.V., and Makarov, V.V., J. Eng. Phys. Thermophys., 2013, vol. 86, p. 798.

8. Bohren, C.F. and Huffman, D.R., Absorption and Scattering of Light by Small Particles, Weinheim: Wiley, 1998; Moscow: Mir, 1986.

9. Van de Hulst, G.C., Light Scattering by Small Particles, New York: Wiley, 1957; Moscow: InLit, 1961.

10. Landsberg, G.S., Optika, ucheb. posobie: dlya vuzov (Optics, a Tutorial for Higher Educ. Inst.), Moscow: Fizmatlit, 2003, 6th ed.

11. Goncharov, V.K., Karaban', V.I., and Kontsevoi, V.L., Kvantovaya Electron., 1991, vol. 18, p. 1231.

12. Goncharov, V.K., Karaban', V.I., Kolesnik, A.V., and Radyuk, I.M., Kvantovaya Electron., 1988, vol. 15, p. 2575.

13. Goncharov, V.K., Kontsevoi, V.L., Puzyrev, M.V., and Smetannikov, A.S., Inzh.-Fiz. Zh., 1994, vol. 66, p. 179.

14. Singh, J.P. and Thakur, S., Laser-Induced Breakdown Spectroscopy, Amsterdam: Elsevier, 2007.

15. Goncharov, V.K., Kozadaev, K.V., and Shchegrikovich, D.V., J. Appl. Spectr., 2013, vol. 80, p. 395.

16. Kozadaev, K.V., Shchegrikovich, D.V., and Makarov, V.V., Elektronika-Info, 2013, no. 1, p. 29.

17. Goncharov, V.K., Kozadaev, K.V., and Shchegrikovich, D.V., J. Eng. Phys. Thermophys., 2011, vol. 84, p. 781 .

18. Goncharov, V.K., Kozadaev, K.V., Makarov, V.V., and Shchegrikovich, D.V., J. Eng. Phys. Thermophys., 2013, vol. 86, p. 798.

19. Goncharov, V.K., Kozadaev, K.V., and Shchegrikovich, D.V., Adv. Opt. Technol., 2012, Art. ID 907292.

20. Kozadaev, K.V., J.Eng.Phys.Thermophys., 2013, vol. 87, p. 704

Translated by A. Seferov 